Japp, K.P., 2002: Wie normal ist Nichtwissen? Replik zu Peter Wehling ,Jenseits des Wissens?“ In: Zeitschrift für Soziologie 31/5 (2002), S. 435-439

Knight, F.H., 1921: Risk, Uncertainty and Profit. http://www.econlib.org/library/Knight/knRUP.html (download 8.10.08)

Luhmann, N., 1992: Ökologie des Nichtwissens. In: Luhmann, N. (Hg.): Beobachtungen der Moderne. Opladen, S. 149-220

March, J.G., Simon, H.A., 1967: Organizations. New York

Merton, R.K., 1936: The Unanticipated Consequences of Purposive Social Action. In: American Sociological Review 6/1 (1936), S. 894-904

Wehling, P., 2001: Jenseits des Wissens? Wissenschaftliches Nichtwissen aus soziologischer Perspektive. In: Zeitschrift für Soziologie 30/6 (2001), S. 465-484

Wehling, P., 2002: Was kann die Soziologie über Nichtwissen wissen? Antwort auf Klaus Japp. In: Zeitschrift für Soziologie 31/5 (2002), S. 440-444

$\langle\gg$

\section{Vertrauen ist gut! Oder ist Kontrolle besser?}

\section{Dieter Klumpp, Herbert Kubicek, Ale- xander Roßnagel, Wolfgang Schulz (Hg.): Informationelles Vertrauen für die Informationsgesellschaft. Berlin, Heidel- berg: Springer Verlag, 2008, 362 S., ISBN 978-3-540-77669-7, € 59,95}

\section{Rezension von Karsten Weber, Opole I Polen, Berlin, Cottbus}

2008 jagte ein Daten-Super-GAU den nächsten: Erst wurde publik, dass die Telekom Geheimdienst spielte und zu Überwachungsmaßnahmen gegriffen hatte, die jeder Idee von Privatsphäre Hohn sprachen. Der nächste Skandal wurde von vielen gar nicht als solcher wahrgenommen, doch müssen die Aussagen einiger Politiker bedenklich stimmen, deren Empfehlung war, dass der Staat statt dezentraler Datensammlungen bei Unternehmen eine große zentrale Datenbank erstellen solle, um Missbrauch zu vermeiden. Ein Schelm, wer Böses dabei denkt. Dann gab es noch Urteile des Bundesverfassungsgerichts zur Onlinedurchsuchung und zur Erfassung von Fahrzeugkennzeichen; die Reak- tionen der abgemahnten Länderinnenminister schwankten zwischen Ignoranz und Sich-bestätigt-Fühlen. Und nun noch der Aufruhr um massive Datendiebstähle, erneut bei der Telekom. Die zweifelhafte Sicherheit von Wahlcomputern, biometrischen Reisepässen und Personalausweisen, die Schönrednerei bezüglich der Ergebnisse des großangelegten BKA-Projekts am Mainzer Hauptbahnhof zur biometrischen Identifikation von Personen oder die Neverending-Story um die Einführung der Gesundheitskarte sind da fast schon Randnotizen. Der Bundesbeauftragte für den Datenschutz mahnt, warnt, wünscht, fordert, droht gar - nur nutzt es nichts. Man möchte vielen Akteuren schlicht den politischen Willen oder die Fähigkeit zur Verbesserung der Situation absprechen. Es wäre also allenthalben Grund genug vorhanden, dass die Bürger der Bundesrepublik Deutschland jegliches Vertrauen in Unternehmen, staatliche Institutionen und Politik verlieren.

\section{Vertrauen}

Die Autorinnen und Autoren des rezensierten Sammelbandes behandeln Vertrauen in Informations- und Kommunikationstechnologien (IuK-Technologien) und in Maßnahmen zum Schutz der Privatsphäre und personenbezogener Daten. Sie konstatieren, dass es bei der Nutzung moderner IuK-Technologie eines „Vertrauens“ bedürfe, weil die Prozesse, die beispielsweise bei der Internetnutzung ablaufen, schwer bis gar nicht zu durchschauen seien. Vertrauen wird im Wesentlichen als Kompensation von Wissen angesehen. Daher ist es überraschend, dass in den Beiträgen empirische Befunde eine geringe Rolle spielen, obwohl es hierzu frei zugängliches Material gibt. Zwar ist das „Special Eurobarometer 196 - Wave 60.0“1 nicht taufrisch, doch da schon seit 2001 Datenschutz und Privatsphäre vom Gesetzgeber eingeschränkt wurden, unterscheidet sich das Erhebungsjahr 2003 bezüglich des Sachverhalts, dass staatliche Institutionen vermehrt Begehrlichkeiten nach personenbezogenen Daten zeigen, nicht wesentlich von heute. Der Studie zufolge seien viele EU-Bürger ,sehr besorgt“ oder „etwas besorgt" darüber, ob ihre Privatsphäre geschützt sei: die Werte in den verschiedenen Ländern schwanken, nimmt man die beiden Ausprägungen zusammen, zwischen 
ca. 40 Prozent und 80 Prozent. Aber nach wie vor vertrauen die EU-Bürger vielen Unternehmensbranchen und Institutionen. Das Wissen der Befragten um Rechte, Bedrohungen und Schutzmaßnahmen bezüglich Datenschutz und Privatsphäre sei jedoch gering; dies bestätige die Annahme, dass Vertrauen fehlendes Wissen zu kompensieren scheint. ${ }^{2}$

\section{Vertrauen durch Technik}

Was bedeutet es aber, Polizei oder Verwaltung Vertrauen hinsichtlich des Umgangs mit personenbezogenen Informationen entgegenzubringen? Herbert Kubicek versucht einige Bedeutungsaspekte von Vertrauen (z.B. in Anlehnung an Niklas Luhmann) herauszuarbeiten und daran anschließend zu fragen, wie Vertrauen technisch hergestellt werden könnte. Rainer Kuhlen wiederum untersucht, wie Vertrauen im Umgang mit IuK-Technologie zerstört wird oder verloren geht, um dann recht schnell auf das Vertrauensmanagement einzugehen. Ortwin Renn und Hans Kastenholz übersetzen Vertrauen kurzerhand mit Glaubwürdigkeit. Dies ist jeweils sehr instruktiv, doch bleibt eine Lücke: Konsultiert man sozialwissenschaftliche Literatur zu Vertrauen, findet man durchweg ein wichtiges Konzept, nämlich Reziprozität. Vertrauen gewähren sich Menschen wechselseitig, aus welchen Gründen auch immer. Technisch orientierte Ansätze gehen hieran jedoch vorbei, da es im Grunde nicht Menschen sind, denen vertraut werden soll, sondern Technik. Das wird beispielsweise bei Dieter Klumpp sichtbar, der Vertrauen übersetzt mit der Erfüllung von Erwartungen hinsichtlich technisch gestützter Dienstleistungen.

Die hohen Vertrauenswerte, die das Special Eurobarometer einigen Branchen und Institutionen attestiert, so meine Vermutung, entstehen dort, wo Menschen noch mit Menschen interagieren; je unpersönlicher jedoch die Interaktion, desto geringer das Vertrauen. Obwohl Rafael Capurro dies nicht explizit ausspricht, kann man seinen Text über Angst und Vertrauen in der Informationsgesellschaft in diese Richtung deuten. Vertrauen bestehe dann, wenn man in der Interaktion mit anderen Menschen einen angemessenen Grad von Sorglosigkeit an den Tag legen könne - man fühlt, weiß oder hofft, dass diese Sorglosigkeit nicht ausgenutzt werde. Angst wiederum sei da, wo man Sorge haben müsse.

Jörg Tauss liefert Gründe dafür, dass wir insbesondere hinsichtlich staatlicher Maßnahmen Sorge um Datenschutz und Privatsphäre haben sollten; eine vergleichbare Zielrichtung verfolgen Klaus Wiegerling et al. mit ihrer Diskussion der Vision des „Ubiquitous Computing“, der Vorstellung, dass Computer nicht mehr als solche erkennbar, sondern in Umgebung und Alltagsgegenstände integriert seien. Dies bedeute letztlich, dass man sich dieser Technologie nicht mehr entziehen könne. Vertrauen spielt in beiden Texten aber eine eher untergeordnete Rolle; tatsächlich nimmt der (explizite) Bezug auf Vertrauen mit einem soziologisch und handlungstheoretisch angereicherten Verständnis im Großen und Ganzen ab, je weiter man in dem Sammelband liest. So ist Verena Metze-Mangolds Text zum Aufbau interkulturellen Vertrauens zwar informativ in Bezug auf internationale Konferenzen und Beschlüsse zur Kulturpolitik und ähnlichen Themen; zur Vertrauensbildung, insbesondere über kulturelle Grenzen hinweg, sagt sie jedoch nichts. Dabei ist dieses Thema von überragender Bedeutung, da eine einheitliche Gesetzgebung für das Internet oder andere IuK-Technologien allenfalls Vision ist - „soft law“ als Ersatz beruht jedoch letztlich auf Vertrauen in regelkonformes Verhalten ohne die Möglichkeit harter Sanktionen. Etwas mehr über Theorie und Praxis zu erfahren, wäre sehr interessant gewesen.

\section{Vertrauen durch vertrauenswürdige Verfahren}

Um Vertrauen herzustellen, müssen die Vertrauensnehmer den Vertrauensgebern überzeugend signalisieren, dass sie vertrauenswürdig sind. Wolfgang Schulz gibt einen kurzen Überblick, wie Vertrauen bzw. Vertrauenswürdigkeit in Informationsgüter hergestellt werden könnte; Ernst Elitz wiederum vertieft dies in Bezug auf (Online-)Journalismus. Die Migration der klassischen Nachrichtenmedien in das Internet bedeutet schließlich nicht nur die Distribution von Informationen über ein anderes Medium; wichtiger ist, die Glaubwürdigkeit des klassischen Journalismus in das neue Medium zu retten. Suchmaschinen bzw. die sie betreibenden Unternehmen, so Marcel Machill und Markus Bei- 
ler in ihrem Beitrag, müssten ebenfalls Glaubwürdigkeit aufbauen - hier bezüglich der Ergebnisse von Suchanfragen und des Umgangs mit Informationen über die Nutzer von Suchmaschinen. Insofern sind Suchmaschinenergebnisse „Vertrauensgüter". Doch zeigt sich erneut, dass unklar ist, wem oder was eigentlich vertraut wird: den Produzenten der Güter, den Produkten selbst, den Produktionsprozessen? Tatsächlich erweitert sich der Kreis noch, denn auch den gelisteten Webseiten und deren Betreibern muss Vertrauen entgegengebracht werden.

Eine ähnliche Situation findet man bei Wikipedia. Doch fraglich ist, ob man die Nutzungshäufigkeit von Suchmaschinen oder Wikipedia, wie es Malte Ziewitz für Letztere tut, als Vertrauensbeweis der Nutzer deuten kann. Meines Erachtens müsste hier den unterstellten Motiven und Einstellungen der Nutzer mehr Skepsis entgegengebracht werden: Viele Studenten beispielsweise nutzten Wikipedia nicht, weil sie sich mit ihr identifizieren, wie Ziewietz schreibt, sondern weil sie nicht gelernt hätten, andere Quellen zu nutzen, oder weil sie $\mathrm{zu}$ bequem seien, in der Bibliothek zu recherchieren. Kurz: Nicht überall, wo Vertrauen draufsteht, ist Vertrauen drin.

Und nicht alles, was als vertrauenswürdig angesehen wird, ist es auch. Dies veranschaulicht Constanze Kurz am brisanten Beispiel der Wahlcomputer, die entgegen allen offiziellen Beteuerungen nachgewiesenermaßen leicht zu manipulieren seien und damit erhebliche Zweifel an den Ergebnissen von Wahlen und letztlich an demokratischen Verfahren aufgeworfen hätten. Hieran zeige sich, dass lang erprobte Prozeduren nicht ohne Weiteres durch computergestützte Verfahren ersetzt werden könnten, wenn nicht die Glaubwürdigkeit der angestrebten Ziele und Ergebnisse infrage gestellt werden solle. Allerdings zeigen Siegfried Hackel und Alexander Roßnagel am Beispiel der langfristigen Aufbewahrung elektronischer Dokumente, dass dieses pessimistische Fazit nicht grundsätzlich gezogen werden müsse, dass aber erheblicher Aufwand notwendig sei, um bewährte Verfahren computergestützt abbilden zu können.

\section{Vertrauen durch Recht und Transparenz}

Tobias Brönneke und Thilo Weichert argumentieren, dass Vertrauen in Geschäftsprozessen
Vertrauenswürdigkeit voraussetze; Datenschutz könne zur Demonstration von Vertrauenswürdigkeit beitragen. Beide Autoren weisen auf, dass der Gesetzgeber Verbraucher nicht ausreichend schütze und diese fast notgedrungen darauf vertrauen müssten - obwohl oftmals keine guten Gründe vorlägen -, dass beispielsweise Unternehmen personenbezogene Daten nicht gegen die Interessen der Betroffenen nutzen. Die Autoren konstatieren hier einen erheblichen Nachholbedarf; die bisherigen Ereignisse des Jahres 2008 geben ihnen dabei Recht. Ähnlich argumentiert Alfred Büllesbach: Vertrauen in den Datenschutz dürfe gerade nicht als Kompensation fehlenden Wissens begriffen werden, sondern müsse dadurch begründet sein, dass die Bürger eines Staates wissen, dass sie Rechte haben und diese auch gut geschützt werden. Die Befunde, die Andreas Mann aus der Praxis des Dialogmarketings mit Bezug auf eigene Untersuchungen sowie umfangreiche Literatur berichtet, sind hierzu eine wichtige Ergänzung, da sich zeigt, dass Vertrauen eine wichtige Komponente für den Geschäftserfolg im Internet darstellt. Erneut wird klar, dass Vertrauen durch Erfahrung begründet sein muss.

Texte zum Jugendschutz erwartet man vielleicht nicht in einem Sammelband zum informationellen Vertrauen, da der Jugendschutz meist als medienpädagogisches Thema angesehen wird. Tatsächlich ist Vertrauen in den Beiträgen von Ben Bachmair, Sabine Frank und Otto Vollmers sowie von Jutta Croll und Herbert Kubicek auch kein zentraler Gegenstand; stattdessen behandeln die Autorinnen und Autoren in erster Linie die Frage, wer im Zusammenhang mit Neuen Medien Kinder und Jugendliche davor schützen könnte, auf jugendgefährdende Inhalte zugreifen zu können.

\section{Mangelndes Vertrauen}

Einigen Beiträgen mangelt es jedoch völlig an Bezügen zu Vertrauen: Bernd Holznagel und Thorsten Ricke liefern eine technische Beschreibung von IPTV (Internet Protocol Television) und eine medienrechtliche Einordnung; Jürgen Malley gibt unter einem eher irreführenden Titel einen Abriss zur Technikfolgenforschung und Politikberatung im Bereich der IuKTechnologie. Fritz Fahrni berichtet über neue Wertschöpfungsketten und Geschäftsmodelle, 
die durch Konvergenz bisher getrennter IuKTechnologien möglich werden; kritische Distanz zu den dargestellten Visionen - mehr stellen die skizzierten Anwendungen meist nicht dar - fehlt dabei. Gerade aber weil Konvergenz Vertrauensprobleme potenziell verschärfen kann, wäre analytische Tiefe wichtig. Frank Leymann wiederum referiert über E-Government in Belgien; im Wesentlichen handelt es sich um einen Sachstandsbericht, dessen Aktualität mangels Quellenangaben nicht überprüft werden kann und der entgegen der Ankündigung in Titel und Einleitung nicht zeigt, dass die E-Government-Lösung in Belgien das Vertrauen der Bürger findet.

\section{Fazit}

Sammelbände kämpfen regelmäßig mit dem Problem der inhaltlichen Heterogenität. Auch der hier rezensierte Band macht da keine Ausnahme. Trotzdem, vielleicht sogar deshalb, sind die meisten Beiträge, gerade auch in dieser Zusammenstellung, höchst instruktiv. Insgesamt wird deutlich, dass Vertrauen ein extrem vielschichtiges Konzept darstellt, das im $\mathrm{Zu}-$ sammenhang mit Technik sowohl zu weiterer, vor allem interdisziplinär ausgerichteter Theoriebildung und Begriffsklärung als auch $\mathrm{zu}$ mehr Empirie auffordert. Die Beiträge bieten hierbei geeignete Ausgangspunkte. Davon abgesehen enthält der Sammelband eine klare Botschaft: Vertrauen ist gut, Kontrolle ist (sehr oft) besser und vor allem dringend notwendig.

\section{Anmerkungen}

1) Die Studie „Data Protection“ der European Opinion Research Group EEIG kann im Internet unter http://ec.europa.eu/public_opinion/archives/ebs/ ebs_196_en.pdf (download 7.10.08) abgerufen werden.

2) Siehe dazu auch: Madden, M.; Fox, S.; Smith, A. et al., 2007: Digital Footprints. Online identity management and search in the age of transparency. Washington; http://www.pewinternet. org/pdfs/PIP_Digital_Footprints.pdf (download 7.10.08)

\section{Nachhaltiges Wirtschaften in Stadt und Region}

\author{
Oscar Reutter (Hg.): Ressourceneffi- \\ zienz - Der neue Reichtum der Städte. \\ Impulse für eine zukunftsfähige Kom- \\ mune. München: oekom verlag, 2007, \\ 271 S., ISBN 978-3865810854, $€ 24,90$
}

\section{Rezension von Volker Stelzer, ITAS}

Leere kommunale Kassen, hohe Arbeitslosigkeit, sinkende Bevölkerungszahlen, wachsende soziale Probleme, starke lokale Umweltbelastungen - überall hört man Klagen über die Schwierigkeiten einer nachhaltigen Stadtentwicklung. In dieser Lage erscheint ein Buch, das vom neuen Reichtum unserer Städte spricht. Wie passt das zusammen?

Für den Herausgeber Oscar Reutter und seine über dreißig Autoren liegt der Schlüssel zur Auflösung dieses Widerspruchs darin, Ressourceneffizienz als strategisches Prinzip beim Entwickeln, Weiterbauen, Erneuern und Verändern der Stadt gezielt einzusetzen. Die These, der in diesem Buch nachgegangen wird, ist, dass Ressourceneffizienz ein wesentlicher Baustein für eine zukunftsfähige Stadtentwicklung ist und für die Gestaltung der technischen Infrastrukturen und das Management des „Konzerns Kommune" nutzbar gemacht werden kann. Hierdurch werden - so die These - neue Chancen für nachhaltiges Wirtschaften in Stadt und Region eröffnet. Die Autoren sehen große Potenziale für einen neuen Reichtum unserer Städte in den kommunalen Infrastrukturen, der Energiewirtschaft, der Wasserwirtschaft, im öffentlichen Personennahverkehr, in der Abfallwirtschaft und im Stoffstrommanagement. Diese ergäben sich aus einem effizienten Umgang mit den natürlichen Ressourcen Energie, Wasser, Material und Fläche. Erschlossen werden könnten sie durch ein aktives, am Prinzip der Ressourceneffizienz orientiertes Stadtmanagement.

In dem vorliegenden Buch wird das Thema vor allem durch Praxiserfahrungen in sechs Handlungsfeldern dargestellt: Der erste Teil widmet sich der Strategie Ressourceneffizienz und den neuen Rahmenbedingungen für eine nachhaltige Stadt- und Regionalentwicklung. 